\title{
Long-lived and Transferable Tumor Immunity in Mice after Targeted Interleukin-2 Therapy
}

\author{
Jürgen C. Becker, ${ }^{\star \ddagger}$ Nissi Varki, ${ }^{\S}$ Stephen D. Gillies, ${ }^{\|}$Koichi Furukawa, ${ }^{\rrbracket}$ and Ralph A. Reisfeld* \\ *The Scripps Research Institute, Department of Immunology, La Jolla, California 92037; ${ }^{*}$ University of Würzburg, Department of \\ Dermatology, 97080 Würzburg, Germany; ${ }^{\S}$ University of California, San Diego, Cancer Center, La Jolla, California 92037; ${ }^{*}$ Fuji \\ ImmunoPharmaceuticals Corp., Lexington, Massachusetts 02173; and "INagasaki University, School of Medicine, Department of \\ Oncology, Nagasaki 852, Japan
}

\begin{abstract}
A major goal of tumor immunotherapy is the induction of tumor-specific $T$ cell responses that are effective in eradicating disseminated tumor, as well as mounting a persistent tumor-protective immunity. We demonstrate here that a genetically engineered fusion protein consisting of human/ mouse chimeric anti-ganglioside $\mathrm{GD}_{2}$ antibody and human interleukin-2 is able to induce eradication of established B78-D14 melanoma metastases in immunocompetent syngeneic C57BL/6J mice. This therapeutic effect is mediated by host immune cells, particularly $\mathrm{CD8}^{+} \mathrm{T}$ cells and is associated with the induction of a long-lived immunity preventing tumor growth in the majority of animals when challenged up to four months later with B78-D14 cells. This effect was tumor-specific, since no cross-protection against syngeneic, ganglioside $\mathrm{GD}_{2}+$ EL-4 thymoma cells was observed. Furthermore, this tumor-specific protection can be transmitted horizontally to naive, syngeneic SCID mice by passive transfer of $\mathrm{CD8}^{+} \mathrm{T}$ lymphocytes derived from immune animals. These results suggest that antibody-targeted delivery of cytokines provides a means to elicit effective immune responses against established tumors in the immunotherapy of neoplastic disease. (J. Clin. Invest. 1996. 98: 2801-2804.) Key words: antibody • cytokine • fusion protein • melanoma $\cdot T$ cell
\end{abstract}

\section{Introduction}

The availability of recombinant immunomodulatory cytokines in pharmacologic quantities and the ability to manipulate these proteins at the genetic level has allowed testing of the effects of these molecules on the host response to cancer in an empiric fashion (1-3). It became obvious that the systemic administration of cytokines to activate any sort of immunological responses fails to account for the fact that these molecules maintain the specificity of immunologic responses partially through their paracrine function (4). Physiologically, systemic concen-

Address correspondence to Dr. Ralph A. Reisfeld, The Scripps Research Institute, $10550 \mathrm{~N}$. Torrey Pines Road, IMM13, La Jolla, CA 92037. Phone: 619-784-8105; FAX: 619-784-2708; E-mail: reisfeld@ scripps.edu

Received for publication 1 July 1996 and accepted in revised form 28 October 1996.

J. Clin. Invest.

(C) The American Society for Clinical Investigation, Inc.

0021-9738/96/12/2801/04 \$2.00

Volume 98, Number 12, December 1996, 2801-2804 trations of most cytokines during an immunologic response are orders of magnitude below the dissociation constant of their receptor, thereby avoiding generalized effects (5). In contrast, systemic administration of cytokines in pharmacologic doses produces high concentrations of these bioactive molecules in the vasculature at sites distant from the tumor, but often at suboptimal levels in the tumor microenvironment (3). A therapeutic approach that conforms with the paracrine nature of cytokines is the ex vivo genetic modification of tumor cells to express them in situ $(4,6)$. When produced by tumors, many of these cytokines induce a local inflammatory response which results in elimination of the injected tumor cells. In some cases, systemic immune responses are generated against challenge with the wild-type parental tumor $(7,8)$. Despite the highly encouraging preclinical data obtained with this approach, the feasibility of its broad clinical application as a cancer therapy seems rather limited by its patient specific nature.

We recently established an alternative approach for cancer therapy which combines effective, local concentrations of cytokines in the tumor microenvironment, low systemic toxicity, and a technically simple modus operandi $(9,10)$. This goal was achieved by construction of fusion proteins consisting of tumor-specific monoclonal antibodies and cytokines; thereby, using the unique targeting ability of antibodies to direct cytokines to the tumor site (11). Antibody-targeted IL2 therapy is able to eradicate established pulmonary, hepatic and subcutaneous melanoma metastases in a syngeneic tumor model. Immunohistochemical analysis, in vivo depletion studies and the use of animals with defined immune defects demonstrated that this eradication of tumor metastases is mediated by host immune cells, particularly CD ${ }^{+} \mathrm{T}$ cells $(9,10)$. Here, we demonstrate that this tumor eradication is associated with induction of a long-lived immunity preventing tumor growth in these animals when challenged up to four months later with the same tumor.

\section{Methods}

Cell lines and reagents. The murine melanoma cell lines, B16 and B78-D14, have been described previously (12). B78-D14 was derived from B16 melanoma cells by transfection with genes coding for $\beta-1,4-$ $\mathrm{N}$-acetylgalactosaminyltransferase and $\alpha-2,8$-sialyltransferase inducing a constitutive expression of the gangliosides $G_{D 2}$ and $G_{D 3}$. B16 melanoma cells were maintained as monolayers in RPMI 1640 medium supplemented with $10 \%$ fetal calf serum and $2 \mathrm{mM}$ L-glutamine and were passaged, as necessary. The culture medium for B78-D14 cells was further supplemented with $400 \mu \mathrm{g}$ G418 and $50 \mu \mathrm{g}$ Hygromycin B per ml.

Mouse/human chimeric antibodies directed against the EGF receptor (ch225) or $\mathrm{G}_{\mathrm{D} 2}$ (ch14.18) were constructed by joining the cDNA for the variable region of the murine antibodies with the constant regions of the $\gamma 1$ heavy chain and the $\kappa$ light chain, as previously 
described (13). The antibody-interleukin-2 fusion proteins, ch225-IL2 and ch14.18-IL2, were constructed by fusion of a synthetic sequence coding for human IL2 to the carboxyl end of the human C $\gamma 1$ gene, as described (11). The fused genes were inserted into the vector phHL2 which encodes for the dihydrofolate reductase gene (14). The resulting expression plasmids were introduced into Sp2/0-Ag14 cells and selected in Dulbecco's modified Eagle's medium supplemented with $10 \%$ fetal bovine serum and $100 \mathrm{nM}$ methotrexate. The fusion proteins were purified over a protein A-Sepharose affinity column. All other antibodies used are commercially available and have been described in detail by the manufacturer (PharMingen, La Jolla, CA).

Animals. C57BL/6J and C57BL/6J scid/scid mice were obtained from The Jackson Laboratory (Bar Harbor, ME) at the age of 4-6 wk. These animals were housed under specific pathogen-free conditions and all experiments were performed according to the National Institutes of Health's guidelines for care and use of laboratory animals.

Experimental lung metastases. Single cell suspension of $5 \times 10^{6}$ tumor cells were injected into the lateral tail vein. To prevent pulmonary embolism caused by injection of tumor cells, mice were anesthetized by halothane inhalation, tumor cells were suspended in $500 \mu \mathrm{l}$ PBS containing $0.1 \%$ BSA and administered i.v. over a period of $60 \mathrm{~s}$. After $7 \mathrm{~d}$, micrometastases were present, disseminated throughout the lungs and invading into the pulmonary alveoli. At day 28 , after tumor cell injection, grossly visible metastases were present on the surface of the organ. Lungs were fixed in Bouin fixative and examined under a low magnification microscope for tumor foci on their surface. Sections from the lungs were stained with hematoxylin/eosin and examined histologically.

Subcutaneous tumors. Tumors were induced by s.c. injection of $5 \times 10^{6}$ tumor cells in RPMI 1640, which resulted in tumors of $\sim 40 \mu \mathrm{l}$ volume within $14 \mathrm{~d}$.

Passive transfer of lymphocytes. Single cell suspensions were prepared by mincing freshly resected spleens into small pieces and passing them through a steel sieve. After washing twice with Hanks balanced salt solution, viable cells were separated on a Ficoll/Hypaque gradient (Nycoprep ${ }^{\mathrm{TM}}$ animal 1.077, Nycomed Pharma, Oslo, Norway) at $600 \mathrm{~g}$ for $20 \mathrm{~min}$, washed twice, counted and resuspended at a concentration of $3 \times 10^{7}$ cells per $\mathrm{ml}$ in PBS containing $0.1 \%$ BSA. Lymphocyte subpopulations were isolated by panning in T-25 flasks coated with covalently immobilized antibodies against CD4 or CD8. Cell suspensions were injected into the lateral tail vein over a period of $60 \mathrm{~s}$. To prevent pulmonary embolism caused by injection of cells, mice were anesthetized by halothane inhalation and the lymphocytes were administered in three aliquots of $1 \times 10^{7}$ cells, each with $2 \mathrm{~h}$ between injections.

Immunohistology. Frozen sections were fixed in cold acetone for $10 \mathrm{~min}$ followed by removal of endogenous peroxidase with $0.03 \%$ $\mathrm{H}_{2} \mathrm{O}_{2}$ and blocking of collagenous elements with $10 \%$ species-specific serum in $1 \%$ BSA/PBS. The antibodies were then overlaid onto serial sections, at predetermined dilutions (usually $20 \mu \mathrm{g} / \mathrm{ml}$ ) and slides were incubated in a humid chamber for $30 \mathrm{~min}$. With PBS washes between every step, a biotinylated link antibody was applied for ten minutes, followed by an enzyme, i.e., either peroxidase or alkaline phosphatase, linked to streptavidin for $10 \mathrm{~min}$. After another wash, the substrate was added and the slides were incubated in the dark for 20 min.

Statistical analysis. The statistical significance of differential findings between experimental groups of animals was determined by Student's $t$ test. The nonparametric Wilcoxon rank sum test was chosen when the data were not amenable for parametric tests, as defined by the David-Pearson-Stephens test. Findings were regarded as significant, if two-tailed $P$ values were $\leq 0.01$.

\section{Results and Discussion}

Since the rejection of IL2-expressing tumor cells in some cases conferred protection against subsequent challenge with the parental tumor $(1,7)$, we tested the hypothesis that tumor eradication mediated by antibody-IL2 fusion proteins may also be associated with the induction of a persistent tumor immunity. Pulmonary metastases were induced by i.v. injection of $5 \times 10^{6}$ B78-D14 murine melanoma cells into syngeneic C57BL/6 mice (12). After one week, when disseminated micrometastases were established, treatment with daily i.v. injections of $8 \mu \mathrm{g}$ of the fusion protein ch14.18-IL2 was initiated and maintained over a period of $7 \mathrm{~d}$. The fusion protein ch14.18-IL2 reacts with the ganglioside $\mathrm{GD}_{2}$, which is expressed on B78-D14 cells. Previous experiments have shown that this treatment cured $>90 \%$ of the animals (10). Six weeks after the initial tumor cell inoculation, these animals were challenged by i.v. injection

Table I. Induction of a Protective Tumor Immunity by ch14.18-IL2 Fusion Protein-mediated Eradication of Established Subcutaneous and Pulmonary Metastases

\begin{tabular}{lllll}
\hline Initial tumor $^{*}$ & \multicolumn{1}{c}{ Treatment } & Interval $[\mathrm{wk}]$ & Challenge $^{\S}$ & \multicolumn{1}{c}{ No of lung foci $^{\mathrm{q}}$} \\
\hline none & none & & B78-D14 & $200,200,>500,>500,>500,>500,>500,>500,>500,>500,>500^{*},>500^{*}$ \\
B78-D14 i.v. & ch14.18-IL2 & 6 & B78-D14 & $0,0,0,0,0,0,0,2,21,56,150,>500$ \\
B78-D14 s.c. & PBS & 6 & B78-D14 & $150,200,>500,>500,>500,>500,>500,>500^{*}$ \\
& cryotherapy & 6 & B78-D14 & $150,150,>500,>500,>500,>500$ \\
& IL2 + ch14.18 & 6 & B78-D14 & $150,>500,>500,>500,>500,>500,>500,>500^{*}$ \\
& ch14.18-IL2 & 4 & B78-D14 & $0,0,0,0,2,26$ \\
& 6 & B78-D14 & $0,0,0,14,43,126$ \\
& 8 & B78-D14 & $0,0,0,4,13,85$ \\
& 12 & B78-D14 & $0,0,0,0,0,128$ \\
& 16 & B78-D14 & $0,0,0,0,9,42$ \\
& 20 & B78-D14 & $0,0,0,12,14,73$ \\
& 8 & EL-4 & $>500^{*},>500^{*},>500^{*},>500^{*},>500^{*},>500^{*}$
\end{tabular}

\footnotetext{
${ }^{\ddagger}$ Primary tumors were induced by intravenous or subcutaneous injection of $5 \times 10^{6}$ B78-D14 melanoma cells in C57BL/6 mice; treatment was started either 7 or $14 \mathrm{~d}$, respectively, thereafter and consisted of daily intravenous injections of PBS, 24,000 IU rIL2 plus $8 \mu \mathrm{g}$ ch14.18 antibody or $8 \mu \mathrm{g}$ ch14.18-IL2 fusion protein. In one group of animals subcutaneous tumors were treated on day 14 with cryotherapy by application of liquid nitrogen for $30 \mathrm{~s}$. Remaining subcutaneous tumors were excised after $4 \mathrm{wk} .{ }^{\S}$ Animals were challenged by intravenous injection of $2.5 \times 10^{6} \mathrm{~B} 78$-D14 cells or $1 \times 10^{6}$ EL-4 thymoma cells at indicated times. " $28 \mathrm{~d}$ after rechallenge animals were killed and evaluated for metastatic disease. Animals that died before that date are marked with an asterisk.
} 
of $2.5 \times 10^{6}$ B78-D14 cells. In 7 of 12 animals, the initial therapy caused complete protection against the tumor challenge and in three animals, the resulting tumor growth was dramatically reduced (Table I). The differences in the number of metastatic pulmonary foci between protected and naive animals were statistically significant with two-tailed $P$ values equal to or less than 0.002 .

Studies of Prehn et al. have convincingly illustrated that the majority of murine tumors are immunogenic, as revealed by vaccination with irradiated tumor cells or by surgical excision of a growing tumor mass $(15,16)$. This inherent immunogenicity markedly complicates the assessment of the immunostimulatory properties of therapeutic manipulations. To account for these problems, we induced subcutaneous tumors by injection of $5 \times 10^{6}$ B78-D14 cells and subjected these to cryotherapy, a combination of recombinant IL2 with the antibody ch14.18 or the ch14.18-IL2 fusion protein. All therapies were started $14 \mathrm{~d}$ after tumor cell inoculation. Both the combination of IL2 with the antibody, as well as the fusion protein were administered i.v. over a period of seven days, once a day, in equivalent amounts. Complete regression of tumors was only observed in those animals that received ch14.18-IL2 with an incidence of $\sim 50 \%$. Remaining tumors were surgically removed four weeks after initial tumor induction. The partial cryotherapeutic removal of the subcutaneous tumors was chosen as one of the controls, since the event of tumor cell destruction may result in an immunizing effect (17). Thus, antigens from destroyed cells may be accessible for uptake and processing by antigen presenting cells (18). However, neither the growth of the subcutaneous tumor itself, nor the presence of tumors altered by cryotherapy or injection of IL2 in combination with ch14.18 caused protection to subsequent challenge with B78-D14 melanoma cells. In contrast, therapy with the tumor-specific fusion protein ch14.18-IL2 completely inhibited the growth of tumors in more than half of the animals (Table I). Time course experiments demonstrated that this protective immunity is longlived, as the same percentage of animals is effectively protected against a challenge with B78-D14 cells over a period of 20 wk after the initial tumor cell inoculation (Table I).

The specificity of this protective immune response was evaluated. Mice, that had previously rejected B78-D14 tumors induced by therapy with ch14.18-IL2 fusion proteins, were challenged by i.v. injection of EL-4 thymoma cells. These cells were chosen, since they are not only syngeneic with C57BL/6 mice, but also share expression of the ganglioside $\mathrm{GD}_{2}$ with B78-D14 cells. It should be noted that B78-D14 cells were generated from B16 melanoma cells by double transfection with genes coding for $\beta-1,4-\mathrm{N}$-acetylgalactosaminyltransferase and $\alpha$-2,8-sialyltransferase inducing a constitutive expression of $\mathrm{GD}_{2}$, which served as target antigen for ch14.18-IL2 (12). As shown in Table I, the rejection of B78-D14 melanoma cells did not induce a cross-protection against the EL-4 thymoma cells.

We have previously shown that $\mathrm{CD}^{+}$cells are essential for the eradication of established micrometastases induced by antibody-IL2 fusion proteins $(9,10)$. To characterize the cells responsible for the protective immunity observed in successfully treated animals, passive lymphocyte transfer studies were performed. Lymphocytes were obtained from spleens of animals which had previously rejected subcutaneous B78-D14 tumors after treatment with ch14.18-IL2. It has been reported that murine antigen-activated/effector $\mathrm{T}$ cells or their progeny are characterized by a Ly-6C+/CD $44^{\text {hi }}$ phenotype $(19,20)$. We ob-
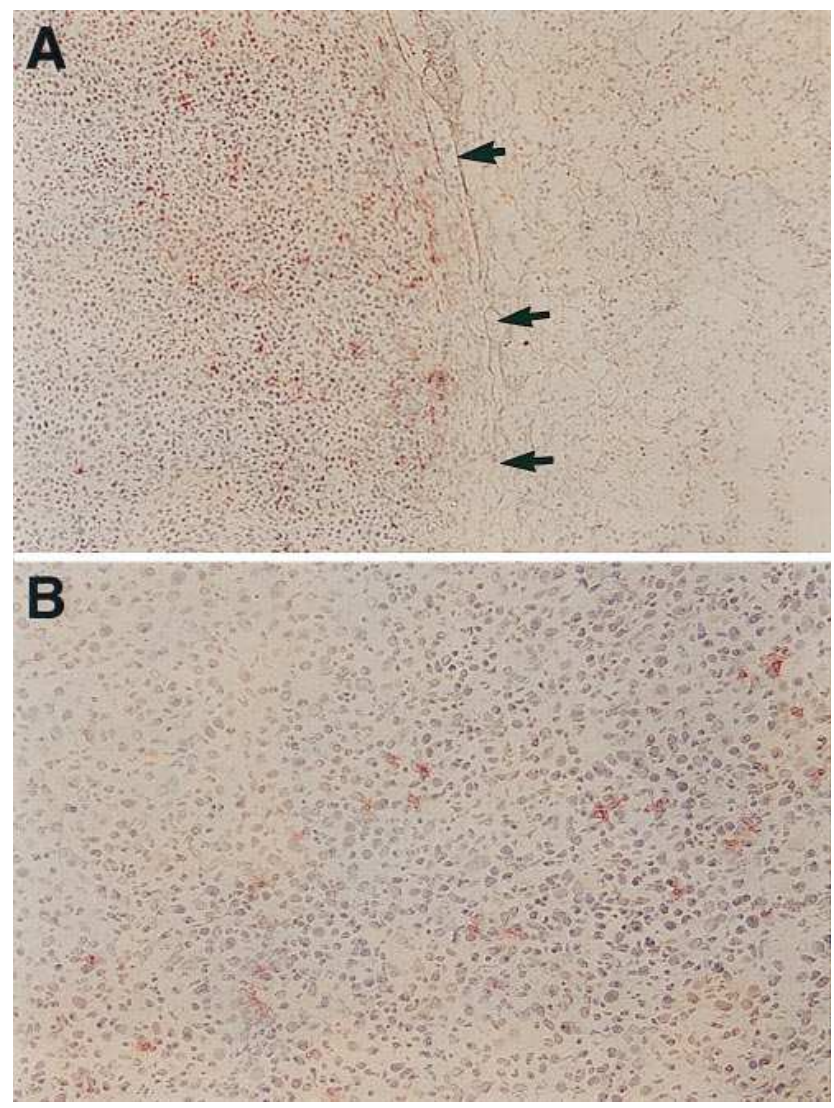

Figure 1. Homing of passively transferred $\mathrm{CD}^{+} \mathrm{T}$ cells to subcutaneous tumors. C57BL/6 scid/scid mice were injected s.c. with $5 \times 10^{6}$ B78-D14 cells. After $18 \mathrm{~d}, 3 \times 10^{7}$ lymphocytes obtained from immunocompetent C57BL/6 mice, that had previously rejected B78-D14 tumors after ch14.18-IL2 treatment, were administered i.v. $24 \mathrm{~h}$ later, $8-\mu \mathrm{m}$ sections of these tumors were prepared and subjected to immunostaining with anti-CD8 antibodies. Arrowheads mark the border between tumor and surrounding tissue. $A, \times 80 ; B, \times 200$.

served that $\sim 30 \%$ of $\mathrm{CD}^{+} \mathrm{T}$ cells used for the transfer experiments indeed expressed this phenotype (data not shown). Transfer of these cells to naive C57BL/6 scid/scid mice $24 \mathrm{~h}$ before challenge with B78-D14 cells confers protection to all animals causing either complete (5/8 animals) or partial (3/8 animals) inhibition of tumor growth (Table II). In contrast, transfer of lymphocytes from naive mice or those suffering from an untreated subcutaneous tumor caused no protection. Enrichment for either $\mathrm{CD}^{+}$or $\mathrm{CD}^{+}{ }^{+} \mathrm{T}$ cells before passive transfer indicated that $\mathrm{CD}^{+} \mathrm{T}$ cells are sufficient to transmit tumor immunity. A second line of evidence demonstrating $\mathrm{CD}^{+}$cells as mediators of this tumor immunity was provided by immunohistologic examination of subcutaneous B78-D14 tumors growing for $18 \mathrm{~d}$ in C57BL/6 scid/scid mice. Tumor samples were obtained $24 \mathrm{~h}$ after passive transfer of lymphocytes isolated from animals which had previously rejected subcutaneous B78-D14 tumors. After this transfer, CD8 ${ }^{+}$, but no $\mathrm{CD}^{+}$cells were detected in the tumor samples (Fig. 1). Interestingly, $\mathrm{CD}^{+}$cells could not be detected in the surrounding tissues adjacent to the tumor demonstrating the specificity of the homing of these cells to the tumor site (21).

The induction of a persistent tumor-specific immunity by 
Table II. Horizontal Transmission of Tumor Immunity by Passive Transfer of Lymphocytes

\begin{tabular}{llll}
\hline \multicolumn{1}{c}{ Donor } & Tumor/therapy & Transfer $^{\S}$ & \multicolumn{1}{c}{ No. of lung foci ${ }^{\sharp}$} \\
\hline None & None & None & $>500,>500,>500^{*},>500^{*}$ \\
C57BL/6 & None & PBL & $>500,>500,>500,>500^{*}$ \\
& B78-D14 s.c. & PBL & $>500,>500,>500,>500^{*}$ \\
& B78-D14 s.c./ & PBL & $0,0,2,45$ \\
& ch14.18-IL2 & & $0,0,0,42$ \\
& & CD4 & $150,200,>500,>500$ \\
& & CD8 & $0,0,0,87$
\end{tabular}

${ }^{\ddagger}$ Subcutaneous tumors were induced by injection of $5 \times 10^{6}$ B78-D14 cells into C57BL/6 mice which served subsequently as donors for lymphocyte transfers. Treatment of these animals with daily intravenous injections of $8 \mu \mathrm{g}$ ch14.18-IL2 fusion protein for seven consecutive days was started on day 14 . Remaining subcutaneous tumors were excised after 4 wk. ${ }^{\S}$ Donor were sacrificed 6 wk after tumor induction and lymphocytes were obtained from pooled spleen cell suspensions. Lymphocyte subpopulations were isolated by panning in T- 25 flasks coated with covalently immobilized antibodies against CD4 or CD8. Either $3 \times 10^{7}$ total lymphocytes; $1 \times 10^{7} \mathrm{CD} 4+$ or $1 \times 10^{7} \mathrm{CD} 8+$ cells were transferred to C57BL/6 scid/scid mice by i.v. injection $24 \mathrm{~h}$ before i.v. challenge with $2.5 \times 10^{6}$ B78-D14 cells. " Animals were killed $28 \mathrm{~d}$ after challenge and evaluated for metastatic disease. Animals that died before that date are marked with an asterisk.

antibody-targeted IL2 therapy, which is mediated by circulating $\mathrm{CD}^{+} \mathrm{T}$ cells, explains the high therapeutic efficacy of this treatment against disseminated, established metastases. In contrast to most other antibody-based therapeutics, that rely on the targeting of all malignant cells $(22,23)$, antibody-mediated cytokine delivery can be effective even if only a small percentage of tumor cells is reached, as these are able to elicit a host immune response. Thus, targeted delivery of cytokines offers a new strategy for tumor immunotherapy that may provide a practicable means to elicit effective T cell-mediated immune responses against established tumors in primary and metastatic disease.

\section{Acknowledgments}

We express our appreciation to James D. Pancook, Ph.D. for his excellent work in the animal surgeries. We also thank Ms. Lynne Kottel for her expert assistance in the preparation of this manuscript.

This work was supported by National Institutes of Health Outstanding Investigator's Award grant CA-42508. J.C. Becker was supported by a training grant of the Deutsche Forschungsgemeinschaft. This is The Scripps Research Institute's manuscript number 9968-IMM.

\section{References}

1. Maas, R.A., H.F.J. Dullens, and W.D. Otter. 1993. IL2 in cancer treatment. Cancer Immunol. Immunother. 36:141-148.

2. Rosenberg, S.A., B.S. Packard, P.M. Aebersold, D. Solomon, S.L. Topalian, S.T. Toy, P. Simon, M.T. Lotze, L.C. Yang, C.A. Seipp, C. Simpson, C. Carter, S. Bock, D. Schwartzentruber, J.P. Wei, and D.E. White. 1988. Use of tumor-infiltrating lymphocytes and interleukin-2 in the immunotherapy of patients with metastatic melanoma. N. Engl. J. Med. 319:1676-1680.

3. Winkelhake, J.L., and S.S. Gauny. 1990. Human recombinant interleukin-2 as an experimental therapeutic. Pharm. Rev. 42:1-64.

4. Pardoll, D.M. 1995. Paracrine cytokine adjuvants in cancer immunotherapy. Annu. Rev. Immunol. 13:399-415.

5. Paul, W.E., and R.A. Seder. 1994. Lymphocyte responses and cytokines. Cell. 76:241-251.

6. Dranoff, G., and R.C. Mulligan. 1995. Gene transfer as cancer treatment. Adv. Immunol. 58:417-454.

7. Zatloukal, K., A. Schneeberger, M. Berger, W. Schmidt, F. Koszik, R. Kutil, M. Cotten, E. Wagner, M. Buschle, G. Maass, E. Payer, S.G. and M.L. Birnstiel. 1995. Elicitation of a systemic and protective anti-melanoma immune response by an IL-2-based vaccine. J. Immunol. 154:3406-3419.

8 Maass, G., W. Schmidt, M. Berger, F. Schilcher, F. Koszik, A. Schneeberger, G. Stingl, M.L. Birstiel, and T. Schweighoffer. 1995. Priming of tumor specific $\mathrm{T}$ cells in the draining lymph nodes after immunization with IL2-secreting tumor cells: three consecutive stages may be required for successful tumor vaccination. Proc. Natl. Acad. Sci. USA. 92:5540-5544.

9. Becker, J.C., N.M. Varki, S.D. Gillies, K. Furukawa, and R.A. Reisfeld. 1996. An antibody-interleukin 2 fusion protein overcomes tumor heterogeneity by induction of a cellular immune response. Proc. Natl. Acad. Sci. USA. 93: 7826-7831.

10. Becker, J.C., J.D. Pancook, S.D. Gillies, K. Furukawa, and R.A. Reisfeld. 1996. T cell mediated eradication of murine metastatic melanoma induced by targeted interleukin 2 therapy. J. Exp. Med. 183:2361-2366.

11. Gillies, S.D., E.B. Reilly, K.-M. Lo, and R.A. Reisfeld. 1992. Antibodytargeted interleukin 2 stimulates T-cell killing of autologous tumor cells. Proc. Nat. Acad. Sci. USA. 89:1428-1432.

12. Haraguchi, M., S. Yamashiro, A. Yamamoto, K. Furukawa, K. Takamiya, K.O. Lloyd, H. Shiku, and K. Furukawa. 1994. Isolation of GD3 synthase gene by expression cloning of GM3 $\alpha-2,8$-sialyltransferase cDNA using antiGD2 monoclonal antibody. Proc. Natl. Acad. Sci. USA. 91:10455-10459.

13. Gillies, S.D., K.-M. Lo, and J. Wesolowski. 1989. High-level expression of chimeric antibodies using adapted cDNA variable region cassettes. J. Immunol. Methods. 125:191-202.

14. Gillies, S.D., D. Young, K.-M. Lo, S.F. Foley, and R.A. Reisfeld. 1991 Expression of genetically engineered immunoconjugates of lymphotoxin and a chimeric anti ganglioside GD2 antibody. Hybridoma. 3:347-356.

15. VanPel, A., and T. Boon. 1982. Protection against a nonimmunogenic mouse leukemia by an immunogenic variant obtained by mutagenesis. Proc. Natl. Acad. Sci. USA. 79:4718-4722.

16. Prehn, R.T., and G.L. Bartlett. 1987. surveillance, latency and the two levels of MCA-induced tumor immunogenicity. Int. J. Cancer. 39:106-110.

17. Prehn, R.T. 1980. Immunotherapy by chemotherapy. INSERM (Inst. Natl. Sante Rech. Med.) Colloq. 97:297-303.

18. Nair, S., A.M.J. Buiting, R.J.D. Rouse, N. VanRooijen, L. Huang, and B.T. Rouse. 1995. Role of macrophages and dentritic cells in primary cytotoxic T cell responses. Int. Immunol. 7:679-688.

19. Sprent, J. 1994. T and B memory cells. Cell. 76:315-322.

20. Walunas, T.L., D.S. Bruce, L. Dustin, D.Y. Loh, and J.A. Bluestone. 1995. Ly-6C is a marker of memory CD8 ${ }^{+}$T cells. J. Immunol. 155:1873-1883.

21. Mackay, C.R. 1991. T Cell memory: the connection between function, phenotype, and migration pathways. Immunol. Today. 12:189-193.

22. George, A.J.T., R.A. Spooner, and A.A. Epenetos. 1994. Applications of monoclonal antibodies in clinical oncology. Immunol. Today. 15:559-561.

23. Ghetie, M.-A., and E.S. Vitetta. 1994. Recent developments in immunotoxin therapy. Curr. Opin. Immunol. 6:707-714. 